\title{
The correlation of pain intensity during local infiltration for spinal anesthesia, and subsequent pain after cesarean section
}

Sasikaan Nimmaanrat, MD, MMed (Pain Mgt)* ${ }^{*}$ Wejpisit Wongwiwattananon, MD $^{*}$ Sirikarn Siripreukpong, MD*, Perinpit Jitmun*

*Department of Anesthesiology, Faculty of Medicine, Prince of Songkla University, Hatyai, Songkhla, Thailand 90110

\section{Abstract}

Background: Local anesthetic infiltration, prior to spinal anesthesia, is generally performed and results in pain upon local injection. This study was conducted to determine the correlation between; pain intensity caused by local anesthetic infiltration in cesarean section patients, and subsequent pain after 24 hours, 1 month and 3 months, postoperatively.

Materials and Methods: This prospective, observational cohort study was performed with 215 patients, scheduled for elective cesarean section. Prior to, a spinal needle insertion, $3 \mathrm{ml}$ of $2 \%$ lidocaine without adrenaline was locally infiltrated via a 24G needle for 4 seconds. The patients were asked to rate their pain intensity, upon local infiltration, by using a verbal numerical rating score (VNRS) from; 0-10, whereas, $0=$ no pain and $10=$ the worst possible pain. The VNRS, at rest and with movement, were evaluated at 24 hours postoperatively as well as at 1 month and 3 months, postoperatively. The correlation was calculated using Spearman's correlation.

Results and Discussion: At 24 hours postoperatively, positive correlation was found between pain intensity of local anesthetic infiltration and acute pain intensity, both at rest $(r=0.554$, $p$-value $<0.001)$ as well as with movement $(r=0.576, p$-value $<0.001)$. Positive correlation was also found for pain at rest $(r=0.296$, $p$-value $<0.001)$ along with movement $(r=0.525$, $p$-value $<0.001)$, at 1 month, postoperatively. At 3 months, positive correlation was found for pain with movement $(r=0.299$, $p$-value $<0.001)$, but there was no statistical significance for pain at rest $(r=0.076, p$-value $=0.273)$.

Conclusions: Statistically, significant correlations have been found between; pain intensity during local infiltration for spinal anesthesia, and both acute, chronic pain after a cesarean section. Local infiltration pain may be used as a predictor for acute and chronic pain, after cesarean section.

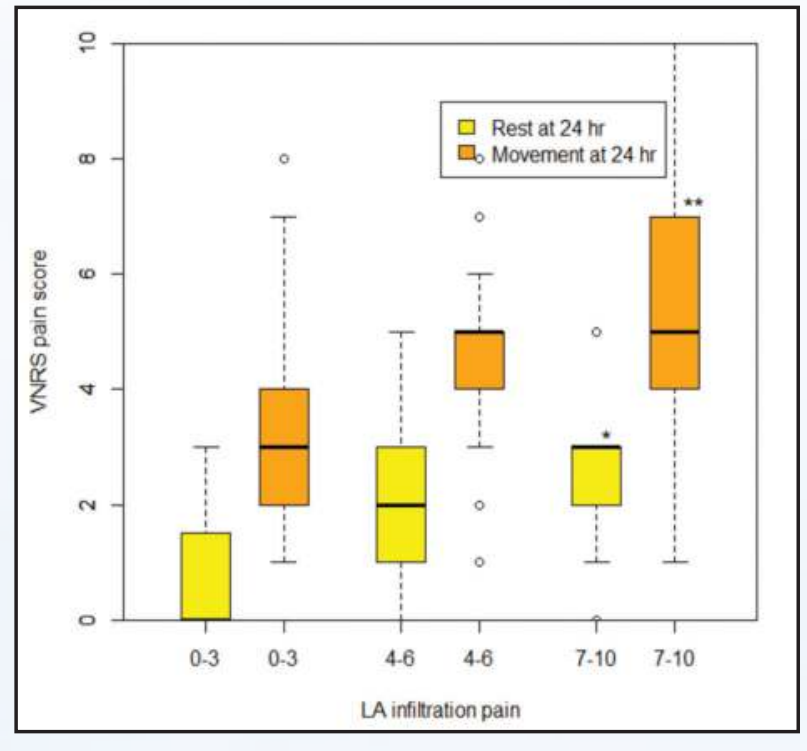

Figure 1: VNRS at rest and with movement at 24 hours

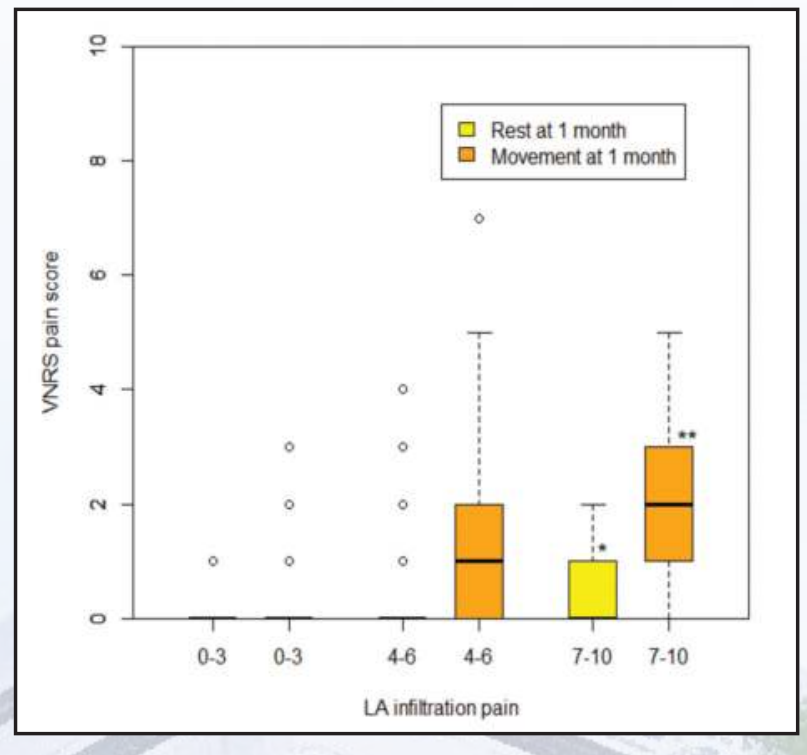

Figure 2: VNRS at rest and with movement at 1 month

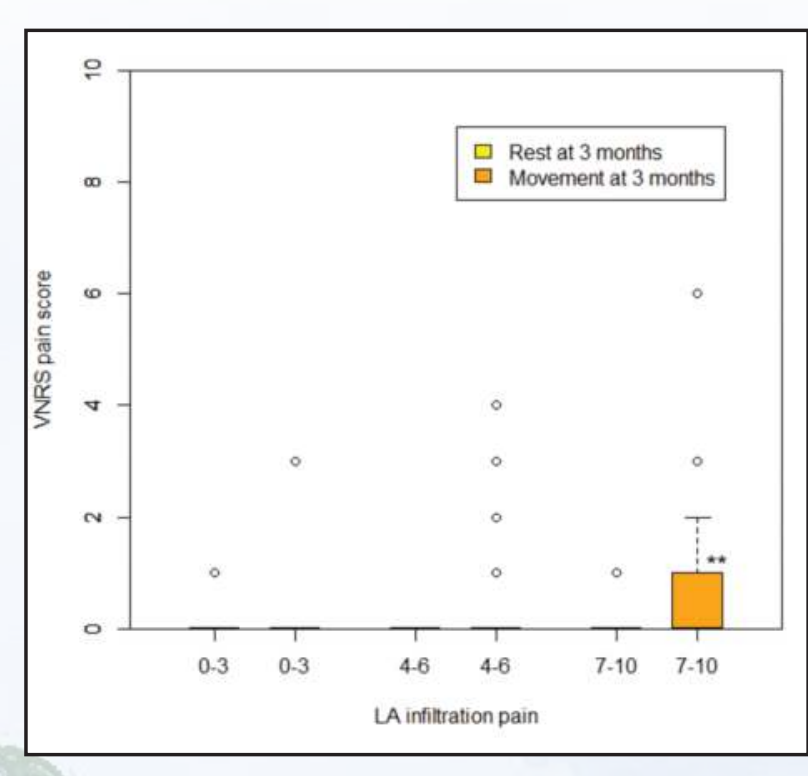

Figure 3: VNRS at rest and with movement at 3 months

Table 1: Correlation coefficient between the pain intensity during local infiltration for spinal anesthesia and pain at rest and with movement after cesarean section at 24 hours, 1 month, and 3 months

\begin{tabular}{|c|c|c|}
\hline & Correlation coefficient & p-value \\
\hline At 24 hours & & $<0.001$ \\
\hline - Pain at rest & 0.554 & $<0.001$ \\
\hline - Pain with movement & 0.576 & $<0.001$ \\
\hline At 1 month & & $<0.001$ \\
\hline - Pain at rest & 0.296 & \\
\hline - Pain with movement & 0.525 & 0.273 \\
\hline At 3 months & & $<0.001$ \\
\hline - Pain at rest & 0.076 & \\
\hline - Pain with movement & 0.299 & \\
\hline
\end{tabular}

